Nonaffine deformation under compression and decompression of a flow-stabilized solid

This content has been downloaded from IOPscience. Please scroll down to see the full text.

J. Stat. Mech. (2016) 084003

(http://iopscience.iop.org/1742-5468/2016/8/084003)

View the table of contents for this issue, or go to the journal homepage for more

Download details:

IP Address: 152.1.53.109

This content was downloaded on 12/08/2016 at 18:48

Please note that terms and conditions apply. 


\title{
Nonaffine deformation under compression and decompression of a flow-stabilized solid
}

\author{
Carlos P Ortiz, Robert Riehn and Karen E Daniels \\ Department of Physics, North Carolina State University, Raleigh, NC 27695, \\ USA \\ E-mail: kdaniel@ncsu.edu
}

Received 31 March 2016, revised 6 June 2016

Accepted for publication 8 July 2016

Published 12 August 2016

Online at stacks.iop.org/JSTAT/2016/084003

doi:10.1088/1742-5468/2016/08/084003

\begin{abstract}
Understanding the particle-scale transition from elastic deformation to plastic flow is central to making predictions about the bulk material properties and response of disordered materials. To address this issue, we perform experiments on flow-stabilized solids composed of micron-scale spheres within a microfluidic channel, in a regime where particle inertia is negligible. Each solid heap exists within a stress field imposed by the flow, and we track the positions of particles in response to single impulses of fluid-driven compression or decompression. We find that the resulting deformation field is well-decomposed into an affine field, with a constant strain profile throughout the solid, and a non-affine field. The magnitude of this non-affine response decays with the distance from the free surface in the long-time limit, suggesting that the distance from jamming plays a significant role in controlling the length scale of plastic flow. Finally, we observe that compressive pulses create more rearrangements than decompressive pulses, an effect that we quantify using the $D_{\min }^{2}$ statistic for non-affine motion. Unexpectedly, the time scale for the compression response is shorter than for decompression at the same strain (but unequal pressure), providing insight into the coupling between deformation and cage-breaking.
\end{abstract}

Keywords: Brownian motion, colloidal glasses, elasticity, plasticity 


\section{Contents}

1. Introduction 2

2. Experimental setup 4

3. Image processing $\quad 5$

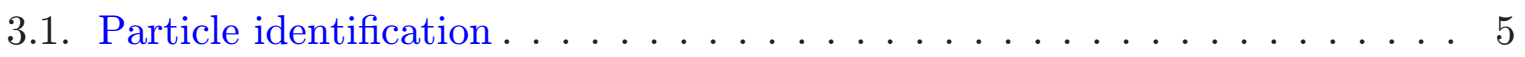

3.2. Total deformation . . . . . . . . . . . . . . . 5

3.3. Short-time particle tracking . . . . . . . . . . . . . . 5

4. Results 6

4.1. Total deformation . . . . . . . . . . . . . . . . 6

4.2. Dynamics of individual particle tracks. . . . . . . . . . . . . 7

4.2.1. Affine deformations . . . . . . . . . . . . . . . . 8

4.2.2. Non-affine deformations . . . . . . . . . . . . . . . . . 9

$\begin{array}{ll}\text { 5. Discussion } & 13\end{array}$

$\begin{array}{ll}\text { Acknowledgments } & 14\end{array}$

$\begin{array}{ll}\text { References } & 14\end{array}$

\section{Introduction}

Understanding how structural rearrangements in disordered solids differ from crystalline solids is central [1-3] to achieving control of material properties such as resistance to flow [4], sound propagation [5], heat capacity [6], and dielectric constants [7]. For large deformations, the microscopic response differs non-perturbatively from the predictions of linear elasticity [8]. Instead of linear deformations, phenomena such as shear banding [9], yielding and plastic rearrangements [10], and non-local effects [11] are present. Recent experiments have explored non-affine deformations in 3D sheared colloidal glasses [12], 3D emulsions [13], and 2D foams [14]. For sufficiently slow deformations, it is an open question whether the flow behavior [15] is controlled by the jamming transition, where moduli vanish as the packing approaches a critical packing fraction [16].

In this paper, we present experiments quantifying the particle-scale deformation of flow-stabilized solids: particle heaps formed under controlled hydrodynamic stress (see figure 1). These quasi-2D heaps are assembled via the slow accumulation of micronscale particles against a barrier within a microchannel, and are found to be stable above Péclet number 1 [17]. For lower Péclet numbers (slower flows) the particles reversibly evaporate away from the solid. We have previously observed that the elastic modulus of the solid is proportional to the confining stress provided by the fluid flow [18]. 
Nonaffine deformation undercompression and decompression of a flow-stabilized solid
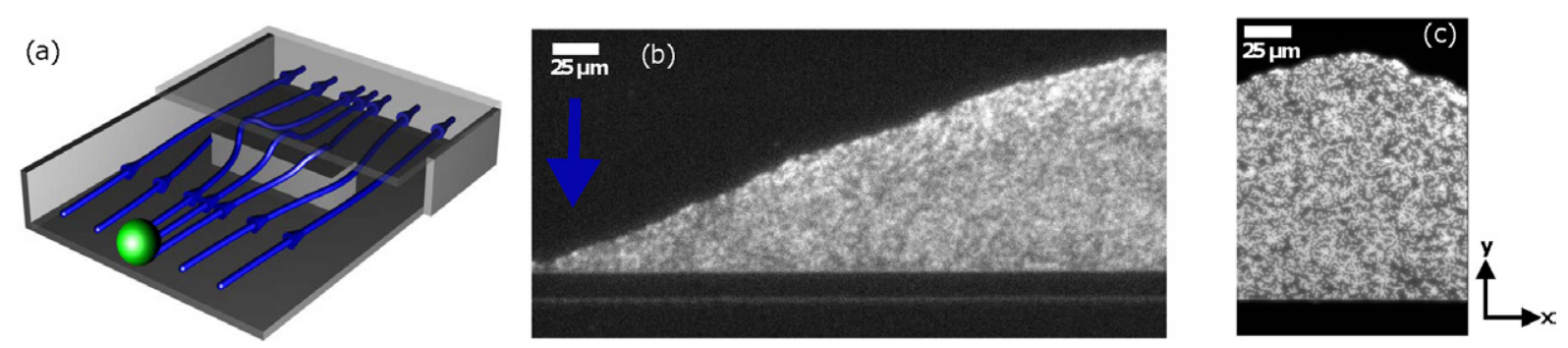

Figure 1. (a) Schematic of channel geometry, not to scale, illustrating barrier with overflow. Streamlines are scaled versions of a calculation solving Stokes' equations on the true device dimensions. This microchannel geometry allows the application of hydrodynamic stresses on particles interacting with the barrier, leading to the formation of microsphere heaps, a flow-stabilized solid. (b) Image of left-half of a heap of bidisperse particles, with the blue arrow showing the direction of fluid flow. (c) Image of central region of a heap of bidisperse particles, used for analysis. Dark regions in the heap in ((b), (c)) are dimly fluorescent $600 \mathrm{~nm}$ particles and bright regions are brightly fluorescent $710 \mathrm{~nm}$ particles. The gray boxes indicate the position of the barrier.

However, the amount of deformation of the solid in response to a flow perturbation is dependent on the sign of the perturbation: for piles prepared under identical conditions, compressions result in smaller strains than decompressions [18]. At the bulk scale, this effect can be understood by considering an excluded volume equation of state, as in thermal systems, under the assumption of locally affine deformations. In this paper, we investigate how the particle-scale dynamics lead to deviations from the excluded volume argument at high deformations.

Our experiments use sterically- and electrostatically-stabilized Brownian microspheres with a short Debye length $(3 \mathrm{~nm})$, so that the net interparticle interaction is well-approximated by a hard-sphere potential except near contact. A bidisperse mixture of particle sizes (5:4 diameter ratio) suppresses the nucleation of crystal domains. Using fluorescence microscopy, we measure the particle-scale deformation fields and characterize the response of the heap under compressive and decompressive loads created by changing the hydrodynamic stress. We characterize the influence of cooperative motions by measuring the degree to which the the deformation field locally deviates from global affine deformations.

The affine (or homogeneous) component of the deformation field is the part that can be described by an affine transformation: rotation, shear, extension, or compression [19]. After identifying the affine component of the deformation, the residual (or inhomogeneous) component is the non-affine deformation. For linearly-elastic materials, only affine deformations are present, but non-affine deformations can arise in structural glasses [20] due to either thermally-driven cage-breaking events [21, 22] or local rearrangements [2]. Here, we quantify two effects: the non-affine rearrangements, and the spatiotemporal dynamics of the response. We observe, as expected [23, 24], that non-affine deformation fields typically exhibit mesoscale correlations. Furthermore, the non-affine deformations are about twice as large for compressive deformations as compared with decompressive deformations of similar size, and happen over a shorter time scale. For both compressive and decompressive deformations, non-affine deformations continue after affine deformations have completed. 


\section{Experimental setup}

Our experiments begin by assembling a microsphere heap by flowing a dilute suspension against a barrier (see figure 1(a)). The microchannel is fabricated to have a height $H=897 \mathrm{~nm}$, higher than the height of a barrier $\left(h_{\mathrm{b}}=694 \mathrm{~nm}\right)$, so that the fluid overflow accumulates particles against the barrier of width $W=512 \mu \mathrm{m}$. The heights are chosen to create a quasi-2D heap, shallow enough to suppress both stacked and non-stacked bilayer phases [25]. The suspension is pumped into the channel by pressurizing a reservoir at the inlet using a piezoelectrically actuated, digital pressure regulator (AirCom PRE1-UA1), at $P_{0}=10 \mathrm{kPa}$ above atmospheric pressure. We collect data once the pile has reached a steady-state (at its maximum size), achieved after about two hours of particle-accumulation. The steady-state heap is $154 \mu \mathrm{m}$ deep ( $30^{\circ}$ angle of repose) and contains approximately 40000 particles, as shown in figure 1(b). The coordinate system takes $\hat{x}$ parallel to the barrier and $\hat{y}$ perpendicular to the barrier, with the origin at center of the barrier; the fluid flow is in the $-\hat{y}$ direction.

The dilute, aqueous suspension is prepared at a areal concentration of $\rho=180 /(100 \mu \mathrm{m})^{2}$ fluorescent microspheres (suspension viewed through microscope). The particles are a bidisperse mixture of equal concentrations of $600 \mathrm{~nm}$ and $710 \mathrm{~nm}$ polystyrene microspheres ( $\approx 6 \%$ polydispersity, elastic modulus $4 \mathrm{GPa}$ from Bangs Laboratories). We use steric and electrostatic stabilization (sulfate functionalized surface with $\zeta$-potential $=-60 \mathrm{mV}$ and coated with Triton X-100 with surface coverage $\approx 1$ molecule/(12 $\AA)^{2}$ ) to provide reversible inter-particle and channel-particle interactions. For long-term stability of the electrostatic interactions, we also hold the suspension $\mathrm{pH}$ constant ( $\mathrm{pH} 5.4$ ), by buffering with citric acid. The suspending fluid is a density-matched aqueous solvent composed of $17 \%(w / v)$ sucrose to suppress segregation and sedimentation effects, important both at the barrier and at the inlet reservoir. Because the total particle brightness scales approximately with the particle volume, and we are working near the diffraction limit, the $600 \mathrm{~nm}$ particles appear dimmer than the $710 \mathrm{~nm}$ particles. This effect aids in tracking the motion of the particles.

We quantify the affine and non-affine deformation due to a pulse of either compression $(\Delta P>0)$ or decompression $(\Delta P<0)$. The decompression pulse results from a change from $10 \mathrm{kPa}$ to $0.5 \mathrm{kPa}$ over a time scale of $10 \mathrm{~ms}$, and the compression pulse returns the pressure to $10 \mathrm{kPa}$. This corresponds to $\Delta P / P_{0}=19$ for the compression pulse and $\Delta P / P_{0}=0.95$ for the decompression pulse. Imaging occurs in two phases. A full view of the initial heap is accessible with a $10 \times$ objective, while experiments quantifying the particle motions require visualizing a zoomed-in region using a $60 \times$ objective with a $4 \times$ beam expander. The image was recorded by a CCD camera with $10 \times 10 \mu \mathrm{m}^{2}$ pixels and an exposure time $36 \mu \mathrm{s}$. For each pulse, we first acquire an image of a region of interest at the center of the heap prior to the pressure change, allowing us to extract the initial configuration of particle positions. Additional images, taken at $27 \mathrm{~Hz}$, characterize the particle-scale response of the heap to the change in pressure. After a wait of $100 \mathrm{~s}$, long enough for particles to settle onto a new, equilibrated configuration, we repeat this process for the compression pulse. 


\section{Image processing}

For either compressive or decompressive pulses, we first compare the initial and final configurations (separated by 100 s), and quantify both the total deformation and the non-affine deformation. Second, using the series of frames immediately following the pulse, we track individual particles to identify non-affine effects on the local scale. Below, these are referred to as 'long-time' and 'short-time' dynamics, and require slightly different image-processing. For the long-time dynamics, the total distance traveled is on the order of a few particle diameters. Therefore, we first subtract the affine deformation before performing particle tracking using the Blair-Dufresne implementation [26] of the Grier-Crocker particle tracking algorithm [27].

\subsection{Particle identification}

Figure 2 summarizes how we obtain particle positions beginning from a raw image. We identify the location of each particle by performing a Wiener deconvolution on the raw image, using a Gaussian approximation to the point-spread function with full-width at half maximum of $540 \mathrm{~nm}$. This value is found to maximize the contrast in the output image, as measured from the ratio of the standard deviation to the mean intensity, but is more effective at locating the large (bright) particles than the small (dim) particles. The resulting deconvolved image allows us to detect the centroid of each particle using Matlab's local extended maxima method.

\subsection{Total deformation}

We estimate the total deformation $\Delta y$ due to a single pulse by making a coarsegrained measurement of the particle displacements between an initial image and a final image. These two images are created by averaging 10 initial images $I_{i}(x, y)=\langle I(x, y, t)\rangle_{t=t_{i} \ldots t_{i}+10 \text { frames }}$ and 10 final images $I_{f}(x, y)=\langle I(x, y, t)\rangle_{t=t_{f} \ldots t_{f}+10 \text { frames }}$. We divide $I_{f}(x, y)$ into horizontal strips of width $2 d$ and compute a cross-correlation with $I_{i}(x, y)$ to determine its displacement, $\Delta y$. We find that the cross-correlation is sharply-peaked function for strips of at least this width. We will show that in section 4.2 an affine deformation consisting of a compression in the $y$-direction is a good approximation of the resulting displacement field.

Due to the large total strains, we perform particle pair matching between initial and final configurations based on particle positions from which the affine deformation has already been subtracted, as described below in section 4.2. After this adjustment, pair identification proceeds as in the one-step particle tracking [26], with the size of the search region selected to correspond to the estimate of the maximum non-affine displacement amplitude, plus an estimate of the error in the affine strain.

\subsection{Short-time particle tracking}

In order to obtain particle trajectories during the full duration of the dynamics, we make several assumptions about the nature of valid trajectories. We limit the displacement per frame to $0.5 \mu \mathrm{m}$; this value is consistent with the affine deformation rate 
Nonaffine deformation undercompression and decompression of a flow-stabilized solid
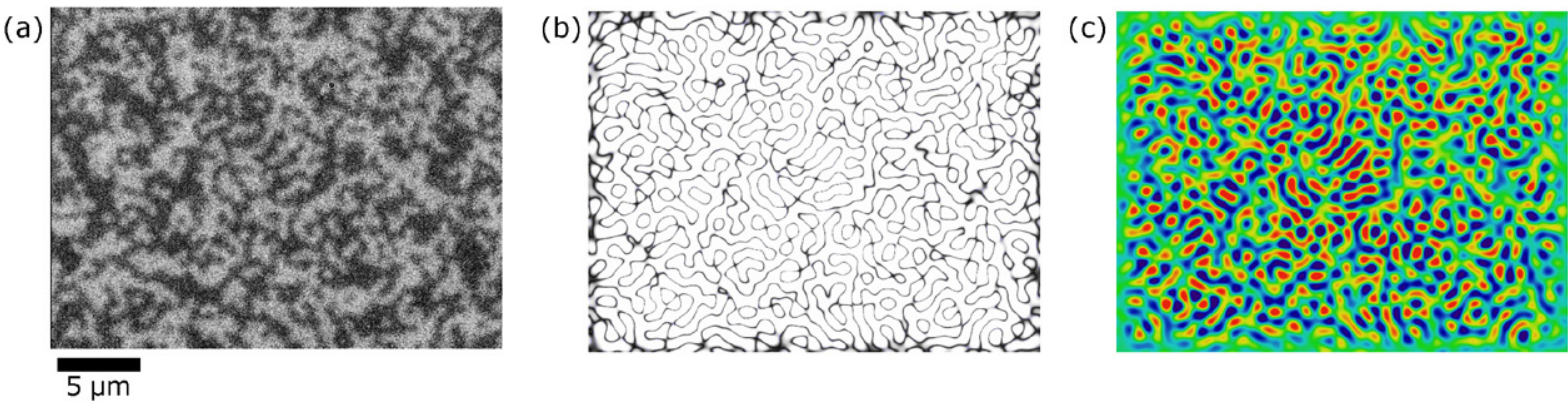

Figure 2. Illustration of image analysis process. (a) Sample micrograph, with grayscale indicating raw data. (b) Edge detection between bright and dark regions. (c) Wiener deconvolved image.

determined above. In addition, we consider a particle's identified size (brightness) in order to either split incorrect trajectories or or reconnect broken trajectories.

\section{Results}

In previous experiments [18], we observed that flow-stabilized solids exhibit a nonlinear stress-strain relationship in which the magnitude of the deformation of the surface of the flow-stabilized solid is well-described by

$$
\frac{\Delta y_{\text {surface }}}{y_{\text {surface }}} \propto\left(\frac{1}{1+\frac{\Delta P}{P_{0}}}-1\right) \text {. }
$$

The success of this description is somewhat surprising, as equation (1) does not contain any information about the distribution of stresses or strains throughout the flowstabilized solid. The stress field within the solid is anticipated to be similar to that in a sedimentation experiment where particles 'on top' of the sedimented material apply some stress on lower layers (in the limit of shallow sediments without side walls). We believe that the success in describing our experiments is due to the universality of the van-der-Waals thermal argument. However, that argument breaks down if non-affine motions occur, and we anticipate that the lower stress at the upstream ('top') surface of the flow-stabilized solid is not fully characterized by the van-der-Waals argument. In the following, we first identify the distributions of particle displacements in the asymptotic long-term limit, before following individual trajectories through compression and decompression. Our particular interest is in the associated particle-scale non-affine motions and their dependence on the sign of $\Delta P$.

\subsection{Total deformation}

We find displacements of individual particles in the heap immediately before and $100 \mathrm{~s}$ after a compressive/decompressive pulse through a two-step analysis. Following the homogeneous strain field assumption from our prior work [18], we first use the crosscorrelation analysis of images before and after deformation to obtain a global estimate 
Nonaffine deformation undercompression and decompression of a flow-stabilized solid
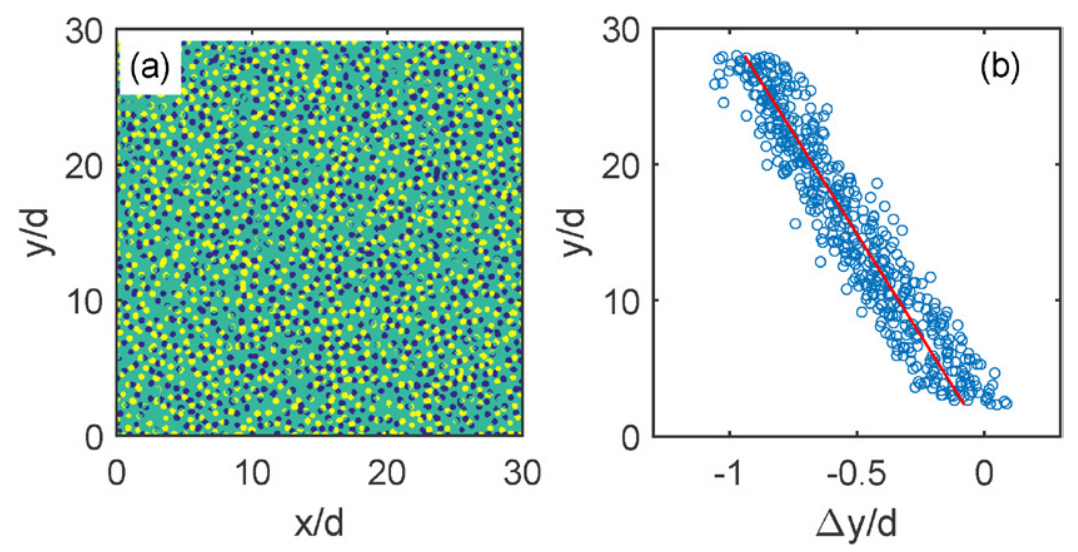

Figure 3. Determination of the long-time affine field from particle tracks for $\Delta P=+9.5 \mathrm{kPa}$. (a) Image difference of blobs with size corresponding to $1 \sigma$ of the position uncertainty in initial (blue) and final (yellow) configuration. (b) The total deformation field plotted only as a function of depth. The dashed line is the associated affine deformation field, given by a linear regression with $\gamma=3.4 \pm 0.2 \%$.

of the affine strain field. We then use the affine transformation identified by the crosscorrelation analysis as a scaffold for the matching of particles in the images before and after deformation. In figure 3(a), we show an example of the particle locations after Wiener deconvolution and prior to finding the centroids, for both $I_{i}$ (red, before compression) and $I_{f}$ (white, after compression). By tracking each centroid, we can plot the local displacement $\Delta y$ as a function of $y$-position within the heap.

As shown in In figure 3(b), the mean behavior is linear, confirming that the overall assumption of an affine deformation was sufficiently accurate. The best fit line to these points provides a measure of the strain: $\Delta y=\gamma_{\infty} y$ with $\gamma_{\infty}=-3.4 \%$. The precision of these measurements is insufficient to estimate the expected higher-order (quadratic) term, although we expect one to be present due to a depth-dependent stress field. The observed linear behavior, combined with equation (1), suggests that the packing fraction is close to invariant along the $y$-direction.

To obtain the non-affine deformation field, we subtract the local affine motion from each displacement vector, as in [2]. Figures 4 and 5 (both compression and decompression) show the total, affine, and non-affine displacement fields, for comparison. Importantly, we observe bands of correlated motions, as expected from [23, 24]. Because the total deformation field is not robust in tracking individual bead pairs over long times, we next examine the short-time dynamics.

\subsection{Dynamics of individual particle tracks}

Using the estimate of strain provided by figure 3(b), we track the fast dynamics arising from a compressive/decompressive pulse. We translate the raw coordinates for particle $p, r_{p}(t)=\left(x_{p}(t), y_{p}(t)\right)$, into the moving reference frame of the affine deformation $\tilde{r}_{p}(t)=r_{p}(t)-\gamma(t) y \hat{y}$, where $\gamma(t)$ is the coarsely estimated affine deformation (see section 3). After tracking in the moving reference frame, we return to the lab reference by applying the inverse of the affine deformation: $r_{p}(t)=\tilde{r}_{p}(t)+\gamma(t) y \hat{y}$, where $\gamma(t)$ is 
(a)

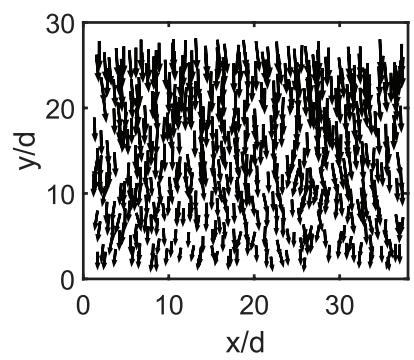

(b)

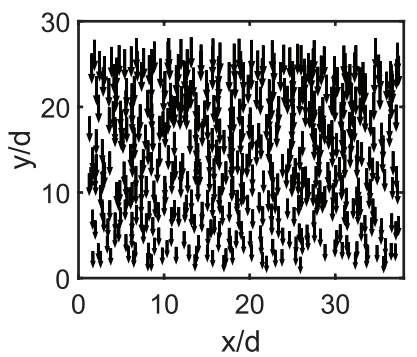

(c)

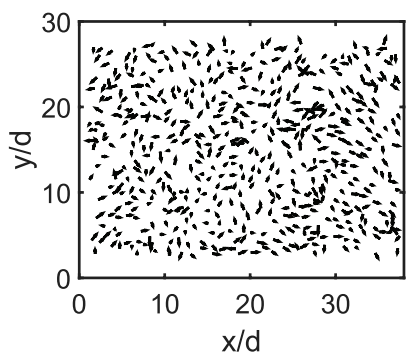

Figure 4. Deformation field for a compression given by a pressure change at the inlet of $\Delta P=+9.5 \mathrm{kPa}$. The axes in both figures are the $x$-position and the $y$-position in units of particle diameters. (a) Full deformation field. (b) Affine deformation field. (c) Non-affine deformation field, magnified by a factor of two.

(a)

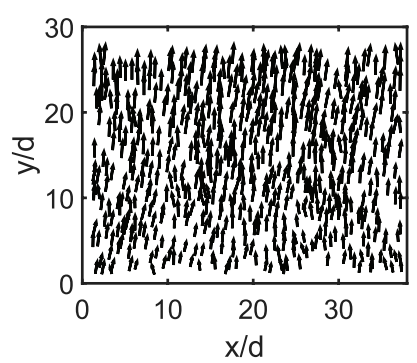

(b)

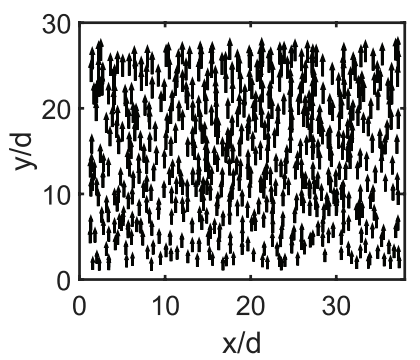

(c)

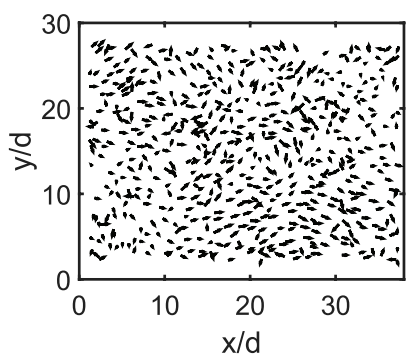

Figure 5. Deformation field for a decompression given by a pressure change at the inlet of $\Delta P=-9.5 \mathrm{kPa}$. The axes in both figures are the $x$-position and the $y$-position in units of particle diameters. (a) Full deformation field. (b) Affine deformation field. (c) Non-affine deformation field, magnified by a factor of two.

the same as used in the forward transformation. The analyses that follow are based on these final coordinates in the lab reference frame.

Sample trajectories are shown in figure 6. While the total deformation field is of the similar magnitude under compression and decompression, we find a more pronounced scrambling of the particle trajectories under compression, as compared to decompression. Below, we quantify both the affine and non-affine contributions to these trajectories.

4.2.1. Affine deformations. Data was binned within strips along the $x$-direction, providing ensembles of particles-dynamics sampled as a function of depth. Figures 7(a) and 8(a) show the mean deformation field $\Delta y$ as a function of $y$-position for compression and decompression, respectively. For both deformation directions, we find an exponential-like asymptotic approach to the final displacement magnitude. The depthdependence of the asymptotic value of $\Delta y$ (figures $7(\mathrm{~b})$ and 8(b)) demonstrates the same linear relationship originally shown in figure $3(\mathrm{~b})$. The resulting slope $\left(\gamma_{\infty}=\Delta y / y\right)$ quantifies the dynamics of affine reorganization. We find a marked difference between decompression (figure 7(c)) and compression (figure 8(c)) in that decompressions are far slower than compressions, and that the strain curves for decompression collapse better onto a single dynamic curve. 

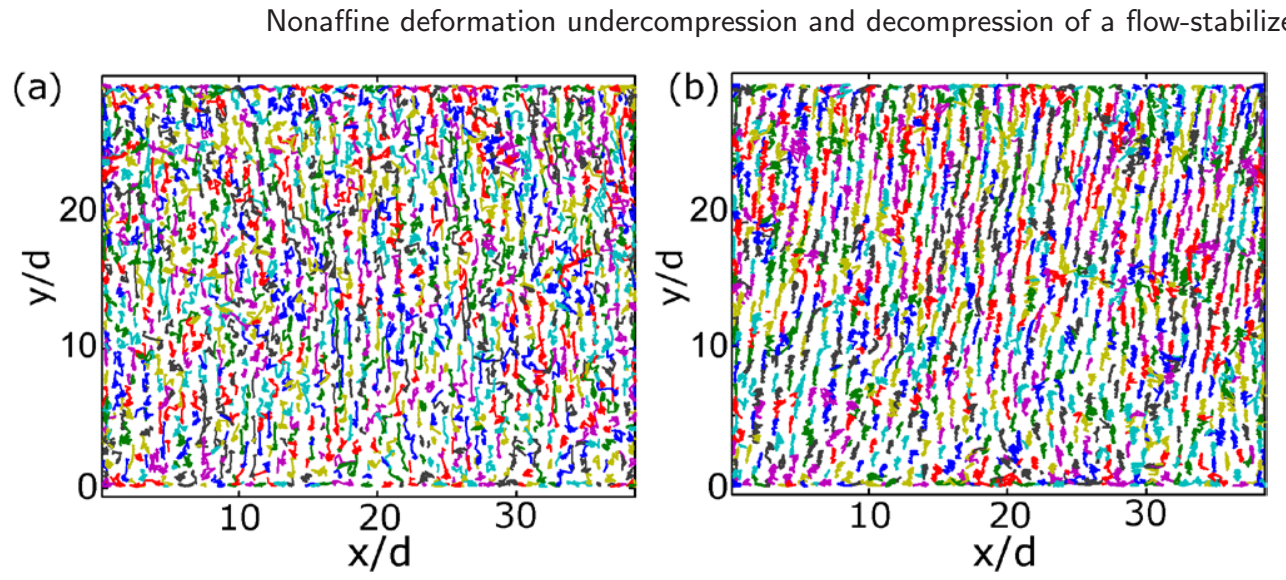

Figure 6. Particle tracks and packing fraction. (a) Particle tracks during compression. Tracks are colored to introduce contrast. (b) Particle tracks during decompression.

To quantify the difference, we make the Ansatz of a single-exponential approach to the asymptotic deformation

$$
\begin{aligned}
& \Delta y(t)=\gamma(t) y \\
& \gamma(t)=\gamma_{\infty}\left(1-\mathrm{e}^{-\frac{\Delta t}{\tau}}\right)
\end{aligned}
$$

where $\Delta y$ is the particle displacement after a time interval $\Delta t, \tau$ is a characteristic time scale of particle rearrangements, $\gamma(t)$ is the time-dependent strain and $\gamma_{\infty}$ is the asymptotic strain. Note that the value $\gamma_{\infty}$ here is a fitting parameter; we find its value to be consistent with the estimate from the long-time dynamics. As shown in both panels (d), this exponential form is a good fit for the decompression pulses with $\tau_{\text {affine, decompression }}=0.28 \pm 0.05 \mathrm{~s}$. For compressive deformations, a single-exponential form is less consistent with the observed dynamics. Instead, there appears to be a twostep process of compression in which the viscous stress increase acts nearly instantaneously throughout the solid, while stresses due to particle-particle contacts propagate at a distinct speed of sound from the immobile barrier on which the solid is formed. Given the two-step nature of the process under compression, we establish an upper bound on the relaxation time scale of $\tau_{\text {affine,compression }}=0.11 \pm 0.05 \mathrm{~s}$.

4.2.2. Non-affine deformations. We identify the non-affine contribution to the deformation field by subtracting the affine portion associated with the best-fit instantaneous value of the strain, which we designate $\gamma(t)$. To quantify the resulting non-affinity, we use the $D_{\min }^{2}$ measure [2] defined by

$$
D_{\min }^{2}(t) \equiv \sum_{\text {neighbors }}\left(\Delta \vec{r}(t)-\gamma(t) \Delta \vec{r}\left(t_{0}\right)\right)^{2} .
$$

Here, $\Delta \vec{r}(t)$ is the set of local displacement vectors connection nearest neighbors, and $t_{0}$ is the time immediately before the pressure step was applied. Figure 9 shows the timeevolution of the non-affine displacement as a function of $y$-position during compression and decompression, respectively. In both graphs, $D_{\min }^{2}$ grows and ultimately saturates. 


\section{Decompression}
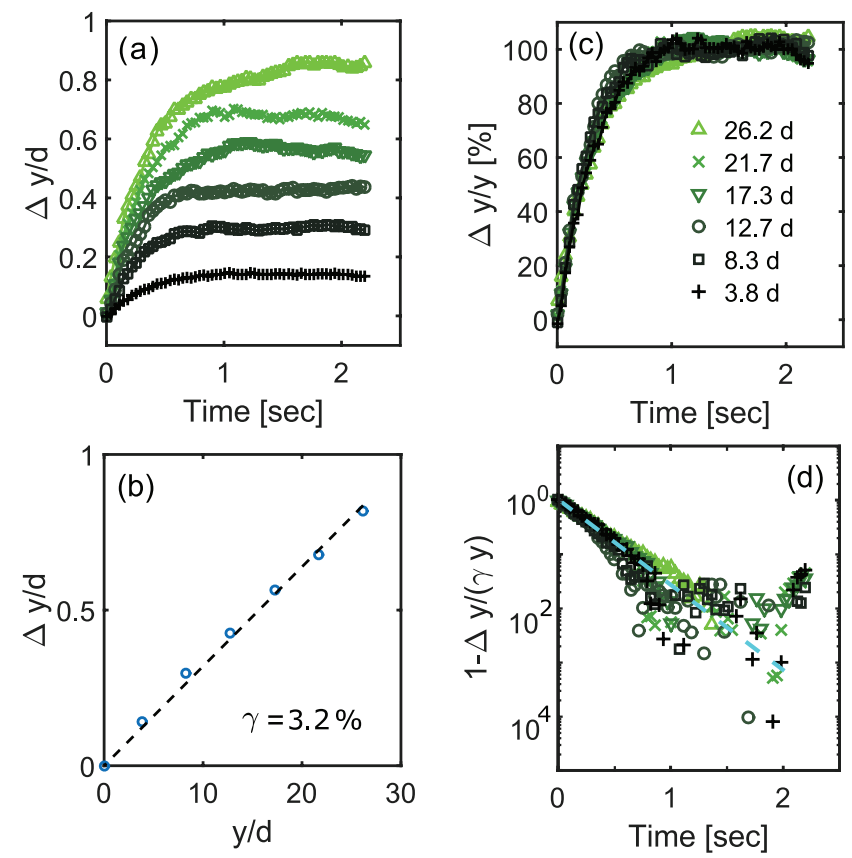

Figure 7. Affine deformation time-series analysis during decompression. (a) Magnitude of correlated displacement field as a function of time at varying distances upstream of the barrier. (b) Long-time displacement amplitude $\Delta y$, as a function of distance upstream of the barrier, both in units of particle diameters. (c) Correlated displacement amplitude normalized by the long-time displacement amplitude. (d) Log-linear plot of growth curves in (c).

Interestingly, the magnitude of the non-affine field scales linearly with depth as demonstrated by the collapse of $D_{\min }^{2} / y^{2}$ data series shown in figure 10 . This a surprising finding in light of the assumed constant strain throughout the flow-stabilized solid. Furthermore, the magnitude of non-affine deformations is approximately twice as large under compression than under decompression at near identical asymptotic strain $\gamma$.

We observe that the growth of $D_{\text {min }}^{2}$ with time is smooth. We are able to determine a characteristic time for the approach to the asymptotic value of $D_{\min }^{2}$ by fitting a single exponential approach, as we did for the affine deformation field. In doing so, we neglect the low background value of $D_{\min }^{2}$ in steady-state flow-stabilized solids arising from Brownian motion. We find $\tau_{\text {non-affine,compression }}=0.36 \pm 0.08 \mathrm{~s}$, and $\tau_{\text {non-affine,decompression }}=0.44 \pm 0.1 \mathrm{~s}$. Therefore, the non-affine field significantly lags the affine field for decompression $\left(\tau_{\text {affine,decompression }}=0.28 \pm 0.05 \mathrm{~s}\right.$ ). For compression, where a single time scale is less well defined, and an upper bound on the affine time scale is $\tau_{\text {affine,compression }}=0.11 \pm 0.05 \mathrm{~s}$, the non-affine field also lags the affine deformation.

To explore the coupling between the non-affine and affine deformation fields, we make a parametric plot of $D_{\min }^{2}$ and $\Delta y$ as a function of time, as explored in figure 11 . As shown in figures 11(a) and (b), the raw growth of $D_{\min }^{2}$ versus displacement magnitude is smooth, following a concave up non-linear relation. Under decompression, the raw growth of $D_{\min }^{2}$ proceeds along a single, characteristic curve for decompression 


\section{Compression}
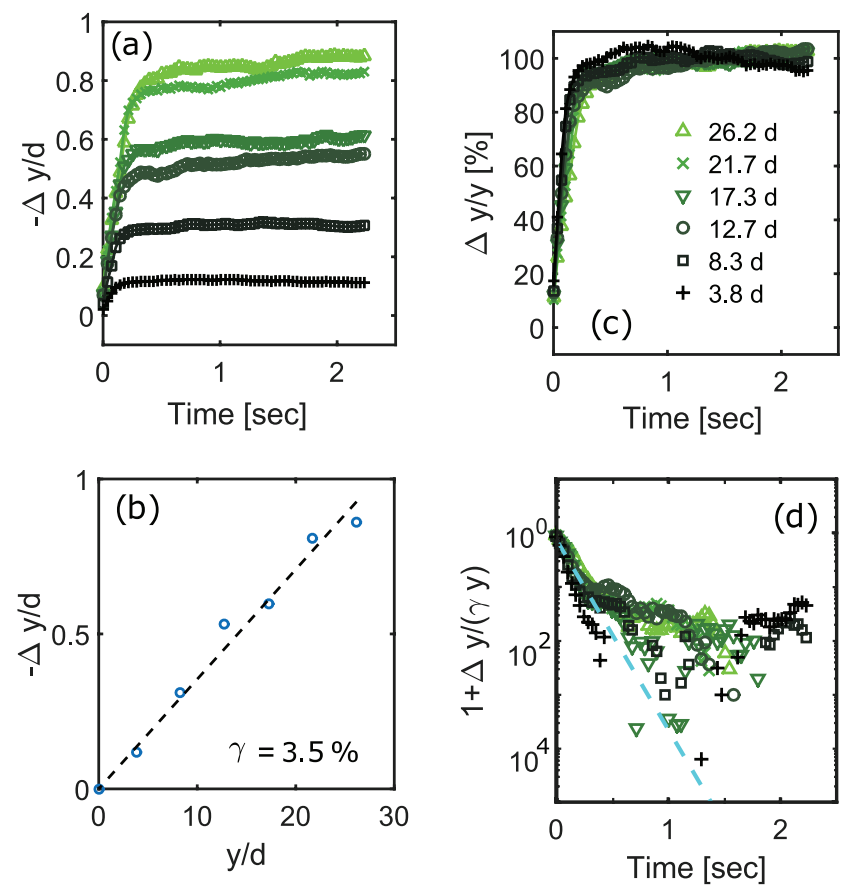

Figure 8. Affine deformation time-series analysis during compression. (a) Magnitude of correlated displacement field as a function of time at varying $y$-positions. (b) Long-time displacement amplitude $\Delta y$, as a function of distance upstream of the barrier, both in units of particle diameters. (c) Correlated displacement amplitude normalized by the long-time displacement amplitude. (d) Log-linear plot of growth curves in (c).
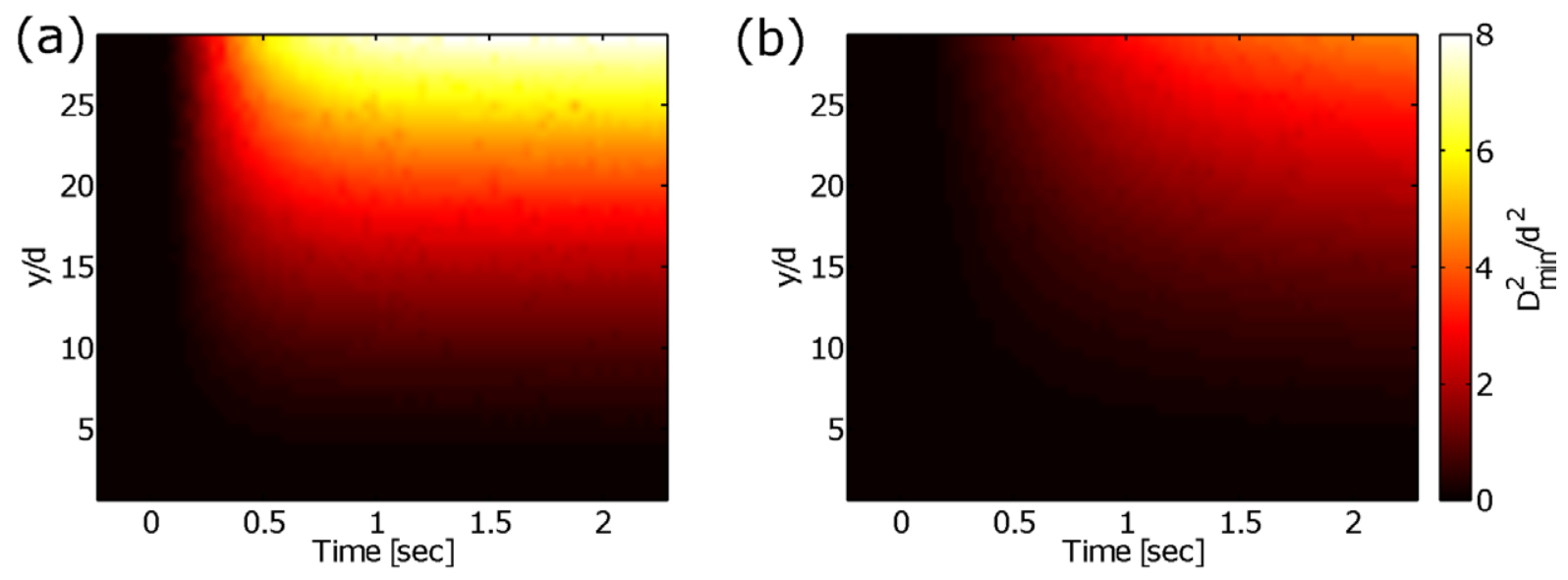

Figure 9. Magnitude of non-affine deformation field $D_{\min }^{2}$ as a function of time and depth for (a) compression and (b) decompression.

(figure 11(b)), but multiple, depth-dependent curves for compression (figure 11(a)). In figures $11(\mathrm{c})$ and (d), we plot $D_{\min }^{2} / y^{2}$, to scale the non-affine deformation field by the depth-dependence suggested in figure 9 , and we find $D_{\min }^{2} / y^{2}$ depends non-linearly on the local-strain $(\Delta y / y)$. Under compression, we find $D_{\min }^{2}$ grows slowly as a function of 
Nonaffine deformation undercompression and decompression of a flow-stabilized solid

Compression
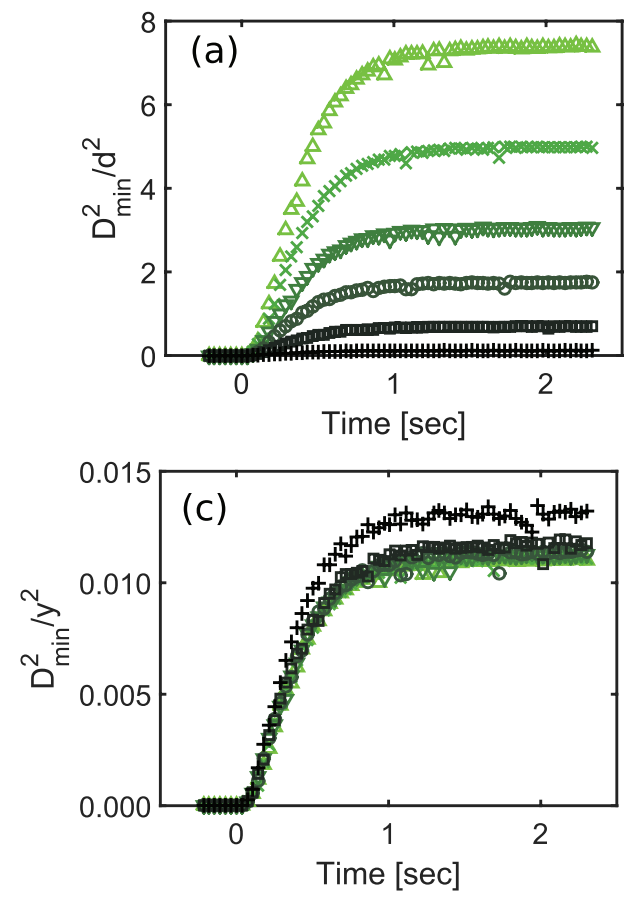

Decompression
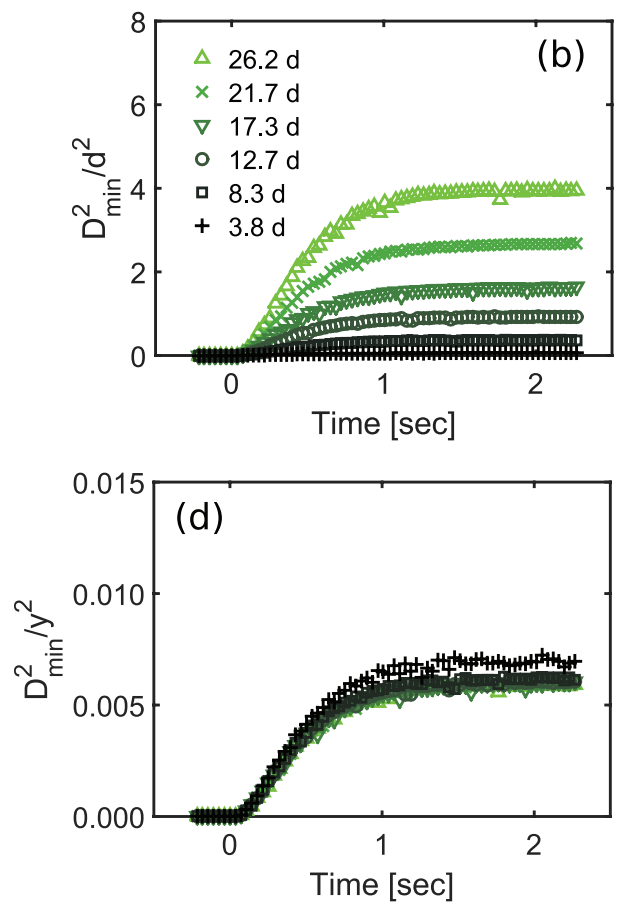

Figure 10. Plots of $D_{\min }^{2}$ as a function of time, with the $y$-axis scaled either by the size of the large particles or the $y$-position at which the non-affine deformation is being probed. The legend in panel (b) applies to all panels. (a) Compression. $D_{\min }^{2}$ scaled by $d^{2}$. (b) Compression. $D_{\min }^{2}$ scaled by $y^{2}$. (c) Decompression. $D_{\min }^{2}$ scaled by $d^{2}$. (d) Decompression. $D_{\min }^{2}$ scaled by $y^{2}$.

strain, but transitions to a rapidly-growing function above a depth-dependent value of the strain figure 11(c). This behavior reflects the two-step nature suggested by studying the temporal evolution of the affine-deformation; when viewed as a function of strain, the behavior in (figure 11(c)) is similar to strain-weakening as seen e.g. in [28]. However, under decompression, $D_{\text {min }}^{2}$ grows smoothly without sudden changes in its rate of change (figure 11(d)). Because figures 11(c) and (d) do not show good collapse, we plot figures $11(\mathrm{e})$ and (f), to determine whether non-affine deformations accumulate via a single-process under decompression, but two processes under compression. Figures $11(\mathrm{e})$ and (f) show $D_{\min }^{2}$ against $(\Delta y)^{2}$, each scaled by its value at $t_{\max }$ to evaluate the depth-dependence of the scaling. Under decompression, this analysis shows that $D_{\min }^{2}$ indeed grows by following a single, depth-independent quadratic curve versus strain. However, under compression, the accumulation of $D_{\min }^{2}$ follows a more complex, strain-dependent set of curves. This asymmetry in the behavior versus driving direction is similar to observations made by [29], who suggested an asymmetry in the response to compression versus decompression and also observed a two-step process under compression, with the second process corresponding to the onset of creep. Our need to wait $100 \mathrm{~s}$ between stress-steps to achieve a new steady state (deformations cease) also suggests the second process may correspond to the onset of creep, but more work is needed to determine the connection between non-affine rearrangements and the onset of creep. 
Nonaffine deformation undercompression and decompression of a flow-stabilized solid
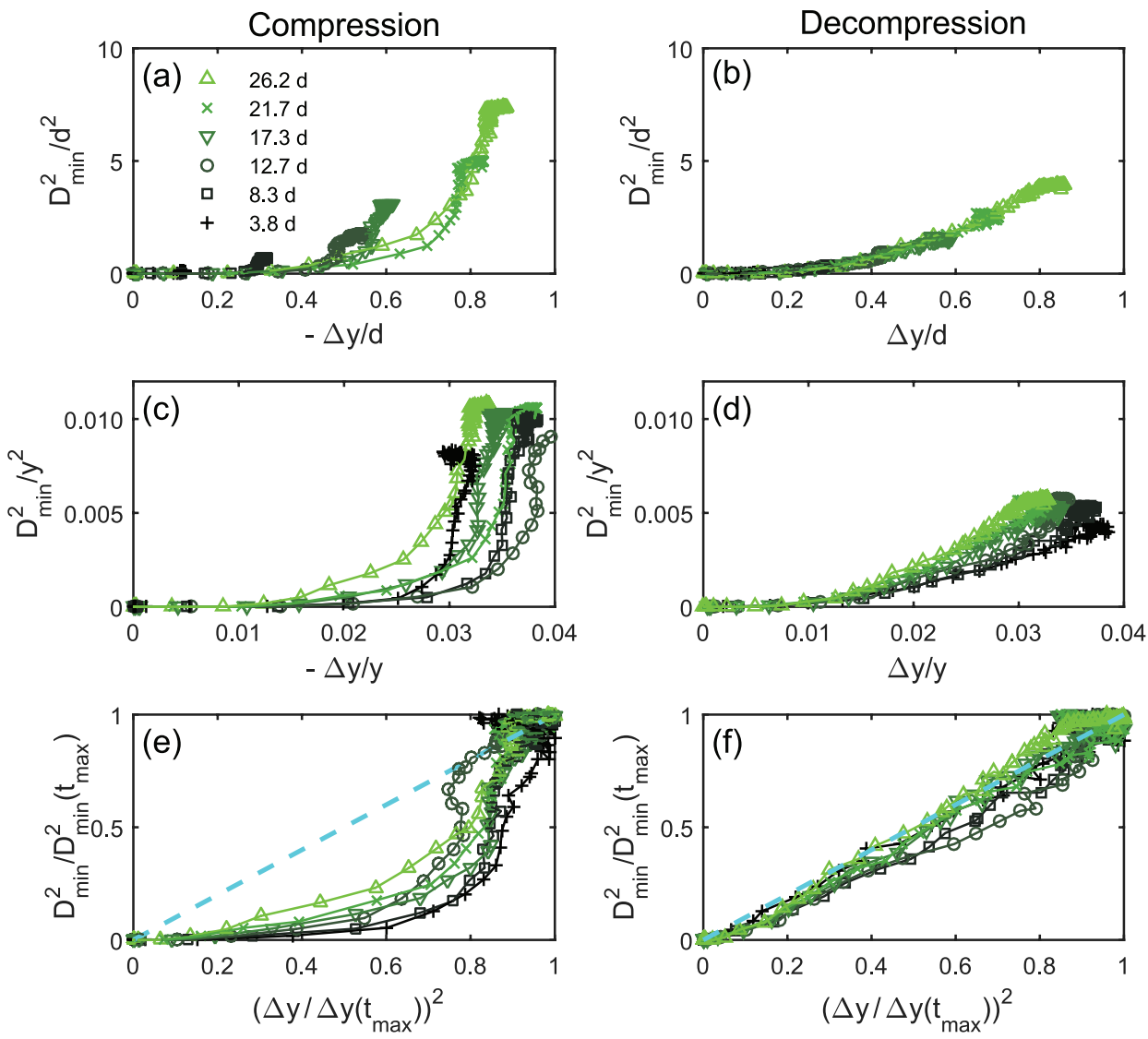

Figure 11. Plots of $D_{\min }^{2}$ as a function of strain, with different scalings. The legend in panel (a) applies to all panels. Left column (panels (a), (c), and (e)) has compression data. Right column (panels (b), (d), and (f)) has decompression data. First row (panels (a) and (b)) is $D_{\min }^{2}$ scaled by $d^{2}$ versus displacement amplitude $\Delta y$. Second row (panels (c) and (d)) is $D_{\min }^{2}$ scaled by $y^{2}$ versus $\Delta y / y$. Third row (panels (e) and (f)) is $D_{\min }^{2}$ versus square displacement amplitude $(\Delta y)^{2}$, each scaled by its value at $t_{\max }$. Dashed line is a guide to the eye along $y=x$.

\section{Discussion}

We have observed particle-scale non-affine motions within flow-stabilized solids, and examined how their spatiotemporal dynamics depend on whether the deformation is compressive or decompressive. We observed the typical swirling regions often associated with non-affine deformations, arising through cooperatively rearranging regions. The magnitude of these effects is nearly twice as large under compression than under decompression, in spite of very similar total strains.

We observe that compressive pulses (large $\Delta P / P_{0}$ ) generate more non-affine deformation, which is able to dissipate the effect of the pulse more quickly. The non-affine fields for both compressive/decompressive deformations occur after similar delays with respect to the affine deformations, which suggests that they are triggered by the affine deformations. In the context of caging behavior, this suggests that the affine deformation distorts the cages provided by the neighboring particles and thereby makes 
Brownian cage-breaking (non-affine deformation) more likely. Remarkably, this is the case even though the strain is approximately the same for decompression and compression. This connection between cage-distortion and the facilitation of cage-breaking events has been suggested by Petekidis [29], but other scenarios are possible as well [20].

In probing the spatial dependence to the magnitude of the non-affine deformations (figure 9), we observe that the degree of non-affinity increases with distance from the barrier. This effect can be rescaled by the position to indicate a universal behavior. The form of this dependence suggests $D_{\min }^{2} \propto \frac{1}{p^{2}} \propto \frac{1}{K}$, for pressure $p$ and modulus $K$ [18]. One interpretation is that the surface of the heap is less rigid (smaller $K$ ), and therefore more prone to undergoing non-affine deformations (higher $D_{\mathrm{min}}^{2}$ ). Similar effects have been observed in numerical simulations [24], where increasingly non-affine displacements are present in proximity to unjamming.

The significance of the above conclusions to soft-matter particle assemblies is to reinforce the centrality of understanding non-affine rearrangements to link bulk properties of the material, such as its modulus and global stability, to local properties about the typical particle geometry and rearrangement timescales. Based on these results, this experimental setup opens the possibility to explore this connection, by studying multiple orders of magnitude of heap sizes, under dynamically tunable interaction potentials and heap geometry, maintaining the ability relate particle-scale rearrangement dynamics to bulk properties. By doing so, it should be possible to determine length and time scales at which localized and collective rearrangements have the greatest impact on bulk properties, and shed light on the general mechanisms by which it is feasible to control the bulk properties of soft matter systems.

\section{Acknowledgments}

We are grateful for support from the National Science Foundation through an NSF Graduate Fellowship, grants DMR-0644743, DMS-0968258, DMR-1121107, MRSEC/ DMR-112090, MRSEC/DMR-1121107, and INSPIRE/EAR-1344280. Research was also supported by US Army Research Office-Division of Earth Materials and Processes grant $64455 \mathrm{EV}$. This work was performed in part at the Cornell NanoScale Facility, a member of the National Nanotechnology Infrastructure Network, which is supported by the National Science Foundation (Grant ECCS-0335765). This work was also performed in part at North Carolina State University facilities: Nanofabrication Facility, Advanced Instrumentation Facility, and Education and Research Laboratory. We also thank one anonymous reviewer for suggesting the parametric plot in figure 11.

\section{References}

[1] Schall P, Weitz D A and Spaepen F 2007 Science 318 1895-9

[2] Falk M L and Langer J S 1998 Phys. Rev. E 57 7192-205

[3] Falk M L and Langer J 2011 Annu. Rev. Condens. Matter Phys. 2 353-73

[4] Brady J F 1993 J. Chem. Phys. 99567

[5] Kriegs H, Petekidis G, Fytas G, Penciu R S, Economou E N and Schofield A B 2004 J. Chem. Phys. $1217849-54$ 
[6] Lubchenko V and Wolynes P G 2007 Annu. Rev. Phys. Chem. 58 235-66

[7] Bradshaw-Hajek B H, Miklavcic S J and White L R 2009 Langmuir 25 1961-9

[8] Bocquet L, Colin A and Ajdari A 2009 Phys. Rev. Lett. 103036001

[9] Hays C C, Kim C P and Johnson W L 2000 Phys. Rev. Lett. 84 2901-4

[10] Hébraud P, Lequeux F, Munch J and Pine D 1997 Phys. Rev. Lett. 78 4657-60

[11] Lu C Y D, Olmsted P D and Ball R C 2000 Phys. Rev. Lett. 84 642-5

[12] Chikkadi V and Schall P 2012 Phys. Rev. E 85031402

[13] Knowlton E D, Pine D J and Cipelletti L 2014 Soft Matter 10 6931-40

[14] Twardos M and Dennin M 2005 Phys. Rev. E 71061401

[15] Lerner E, Düring G and Wyart M 2012 Proc. Natl Acad. Sci. USA 109 4798-803

[16] Liu A J and Nagel S R 2010 Annu. Rev. Condens. Matter Phys. $1347-69$

[17] Ortiz C P, Riehn R and Daniels K E 2013 Soft Matter 9543

[18] Ortiz C P, Daniels K E and Riehn R 2014 Phys. Rev. E 90022304

[19] Wu H C 2005 Continuum Mechanics and Plasticity (London: Chapman and Hall)

[20] Pica Ciamarra M, Pastore R and Coniglio A 2016 Soft Matter 1210

[21] Weeks E and Weitz D 2002 Phys. Rev. Lett. 89095704

[22] Pastore R, Pica Ciamarra M, Pesce G and Sasso A 2015 Connecting short and long time dynamics in hardsphere-like colloidal glasses Soft Matter 11 622-6

[23] Léonforte F, Tanguy A, Wittmer J and Barrat J L 2006 Phys. Rev. Lett. 97055501

[24] Ellenbroek W G, Hecke M V and Saarloos W V 2009 Phys. Rev. E 8061307

[25] Van Winkle D and Murray C 1986 Phys. Rev. A 34 562-73

[26] Blair D and Dufresne E The matlab particle tracking code repository http://physics.georgetown.edu/matlab/

[27] Crocker J C and Grier D G 1996 J. Colloid Interface Sci. 179 298-310

[28] Dang M T, Denisov D, Struth B, Zaccone A and Schall P 2016 Eur. Phys. J. E 391

[29] Petekidis G, Vlassopoulos D and Pusey P N 2004 J. Phys.: Condens. Matter 16 S3955 